\title{
Carboxylate-Based Receptors for the Recognition of Carbohydrates in Organic and Aqueous Media
}

\author{
Monika Mazik* and Hüseyin Cavga
}

1. Syntheses of the the compounds $9, \mathbf{1 0}, \mathbf{1 1 a}$ and $\mathbf{1 1 b}$.

Page

2. ${ }^{1} \mathrm{H}$ NMR titration of receptor $\mathbf{1 1 b}$ with $\beta$-glucopyranoside $\mathbf{1 2 b}$ in $\mathrm{CDCl}_{3}$ (chemical shifts of the $\mathrm{CH}_{3}$ and the pyridine $\mathrm{CH}$ resonances).

3. $\quad{ }^{1} \mathrm{H}$ NMR titration of receptor $\mathbf{1 1 b}$ with $\alpha$-glucopyranoside $\mathbf{1 3}$ in $\mathrm{CDCl}_{3}$.

4. ${ }^{1} \mathrm{H}$ NMR titration of receptor 9 with $\alpha$-glucopyranoside 13 in $\mathrm{CDCl}_{3}$ (chemical shifts of the $\mathrm{NH}, \mathrm{CH}_{2}$ and $\mathrm{CH}_{3}$ resonances).

5. ${ }^{1} \mathrm{H}$ NMR titration of receptor $\mathbf{1 1 a}$ with cellobiose $\mathbf{1 4}$ in water (chemical shifts of the $\mathrm{CH}_{3}$ resonances).

6. $\quad{ }^{1} \mathrm{H}$ and ${ }^{13} \mathrm{C}$ NMR spectra of compounds $9, \mathbf{1 0}, \mathbf{1 1 a}$ and $\mathbf{1 1 b}$.

7. Crystal data for compound $\mathbf{9}$ (Tables of atomic coordinates and equivalent isotropic displacement parameters, bond lengths and angles, anisotropic displacement parameters, the anisotropic displacement factor, hydrogen coordinates and isotropic displacement parameters 
1. Syntheses of the compounds $9, \mathbf{1 0}, \mathbf{1 1 a}$ and $\mathbf{1 1 b}$.

${ }^{1} \mathrm{H}$ and ${ }^{13} \mathrm{C}$ NMR spectra were measured using a 400 and $500 \mathrm{MHz}$ spectrometer; chemical shifts are reported in ppm downfield to TMS as internal standard. Analytical TLC was carried out on silica gel $60 \mathrm{~F}_{254}$ plates employing a methanol-chloroform 1:7 (v/v) as the mobile phase. Melting points are uncorrected.

\section{1-[Bis(N,N-diethoxycarbonylmethyl)aminomethyl]-3,5-bis[(6-methyl-pyridin-2-yl)-}

aminomethyl]-2,4,6-trimethyl-benzene (9): A mixture of 1,3,5-tris(bromomethyl)-2,4,6trimethyl-benzene ${ }^{12}(3.00 \mathrm{~g}, 7.50 \mathrm{mmol}), \mathrm{K}_{2} \mathrm{CO}_{3}(3.15 \mathrm{~g}, 22.50 \mathrm{mmol})$ and iminodiacetic acid diethyl ester $(1.41 \mathrm{~g}, 7.50 \mathrm{mmol})$ in $\mathrm{CH}_{3} \mathrm{CN}(120 \mathrm{~mL})$ was stirred at room temperature for 45 min. Then 2-amino-6-methyl-pyridine (1.62 g, $15 \mathrm{mmol})$ in THF (50 mL) were added, and the mixture was stirred at room temperature for $48 \mathrm{~h}$. After filtration and evaporation of solvents, the crude product was purified by column chromatography (ethyl acetate/toluene 1:1 v/v). Yield 33\%. M.p. $116-118{ }^{\circ} \mathrm{C} .{ }^{1} \mathrm{H}-\mathrm{NMR}\left(400 \mathrm{MHz}, \mathrm{CDCl}_{3}\right): \delta=7.36(\mathrm{t}, 2 \mathrm{H}, J=7.8$ $\mathrm{Hz}), 6.47(\mathrm{~d}, 2 \mathrm{H}, J=7.1 \mathrm{~Hz}), 6.26(\mathrm{~d}, 2 \mathrm{H}, J=8.3 \mathrm{~Hz}), 4.38(\mathrm{~d}, 4 \mathrm{H}, J=4.3 \mathrm{~Hz}), 4.23(\mathrm{t}, 2 \mathrm{H}, J$ $=4.3 \mathrm{~Hz}), 4.12(\mathrm{q}, 4 \mathrm{H}, J=7.1 \mathrm{~Hz}), 4.04(\mathrm{~s}, 2 \mathrm{H}), 3.51(\mathrm{~s}, 4 \mathrm{H}), 2.44(\mathrm{~s}, 6 \mathrm{H}), 2.38(\mathrm{~s}, 9 \mathrm{H}) .1 .25$ $(\mathrm{t}, 6 \mathrm{H}, J=7.1 \mathrm{~Hz}) .{ }^{13} \mathrm{C}-\mathrm{NMR}\left(100 \mathrm{MHz}, \mathrm{CDCl}_{3}\right): \delta=171.4,158.2,157.1,138.0,137.8$, 136.6, 133.4, 132.8, 112.2, 102.9, 60.3, 53.1, 52.3, 41.8, 24.4, 15.9, 14.2. HR-MS calcd for $\mathrm{C}_{32} \mathrm{H}_{43} \mathrm{~N}_{5} \mathrm{O}_{4}$ 561.3313; found: 561.3315. $R_{f=} 0.74$ (methanol-chloroform 1:7 v/v).

Diacid 10: Diester 9 (103 mg, $0.18 \mathrm{mmol}$ ) was dissolved in a solution containing THF (10 $\mathrm{mL}), \mathrm{MeOH}(10 \mathrm{~mL}), \mathrm{H}_{2} \mathrm{O}(10 \mathrm{~mL})$ and $\mathrm{NaOH}(16 \mathrm{mg}, 0.40 \mathrm{mmol})$. The solution was stirred at room temperature for $15 \mathrm{~h}$. The organic solvents were removed under reduced pressure and the water phase was acidified with $5 \% \mathrm{HCl}$. The solution was evaporated to dryness and the residue was suspended in $\mathrm{EtOH}$. After filtration and evaporation of $\mathrm{EtOH}$, the obtained 
powder was dried, and then suspended in $\mathrm{CHCl}_{3}$ containing $\mathrm{NEt}_{3}$. This mixture was stirred at room temperature for $45 \mathrm{~min}$ and then evaporated to dryness. The residue was washed with small amounts of water (separation of triethylamine hydrochloride) and dried to obtaine 9 as a white powder.

Yield 93\%. M.p. $161-162{ }^{\circ} \mathrm{C} .{ }^{1} \mathrm{H}-\mathrm{NMR}$ (400 MHz, DMSO): $\delta=7.24$ (t, 2H, $J=7.8 \mathrm{~Hz}$ ), 6.36 (d, 2H, $J=7.1 \mathrm{~Hz}), 6.23(\mathrm{~d}, 2 \mathrm{H}, J=8.3 \mathrm{~Hz}), 4.39(\mathrm{~d}, 4 \mathrm{H}, J=4.3 \mathrm{~Hz}), 3.95(\mathrm{~s}, 2 \mathrm{H}), 3.41(\mathrm{~s}$, 4H), 2.44 (s, 6H), 2.34 (s, 3H), 2.30 (s, 6H). ${ }^{13} \mathrm{C}-\mathrm{NMR}(100 \mathrm{MHz}, \mathrm{DMSO}): \delta=171.7,158.1$, 155.3, 137.1, 136.8, 136.3, 133.6, 132.1, 110.3, 105.5, 52.6, 51.7, 40.4, 24.1, 15.7. HR-MS calcd for $\mathrm{C}_{28} \mathrm{H}_{33} \mathrm{~N}_{5} \mathrm{O}_{3}$ [M- $\left.\mathrm{H}_{2} \mathrm{O}\right]$ 487.2591; found: 487.2584. $R_{f}=0.20$ (methanol-chloroform 1:7 v/v). Sodium salt 11a: $\mathrm{HR}-\mathrm{MS}$ calcd for $\mathrm{C}_{28} \mathrm{H}_{34} \mathrm{~N}_{5} \mathrm{Na}_{2} \mathrm{O}_{4}[\mathrm{M}+\mathrm{H}]^{+}$550.2406; found: 550.2403

Bis(tetramethylammonium) salt 11b: Two equiv. of a $0.1 \mathrm{M}$ solution of $\mathrm{Me}_{4} \mathrm{NOH}$ in methanol/2-propanol were added to the diacid in methanol. The resulting solution was stirred, evaporated to dryness under reduced pressure, and the solid salt was dried in vacuo. HR-MS calcd for $\mathrm{C}_{28} \mathrm{H}_{34} \mathrm{~N}_{5} \mathrm{O}_{4}\left[\mathrm{M}-\left(\mathrm{NMe}_{4}\right)_{2}+\mathrm{H}\right]^{-}$504.2610; found: 504.2611.

Binding Studies. ${ }^{1} \mathrm{H}$ NMR titrations were performed at $298 \mathrm{~K}$ in $\mathrm{CDCl}_{3}$ stored over activated molecular sieves and deacidified with $\mathrm{Al}_{2} \mathrm{O}_{3}$ (for each titration 10-20 samples were prepared). The titration data were analyzed by nonlinear regression analysis using the Hostest 5.6 program. ${ }^{13}$ For each system at least 3 titrations were carried out. The used concentration ranges are given in the legend of Figures 2-7 and S1-S4. 
2. ${ }^{1} \mathrm{H}$ NMR titration of $\mathbf{1 1 b}$ with $\beta$-glucopyranoside $\mathbf{1 2 b}$ in $\mathrm{CDCl}_{3}$ (chemical shifts of the $\mathrm{CH}_{3}$ and the pyridine $\mathrm{CH}$ resonances).

a)

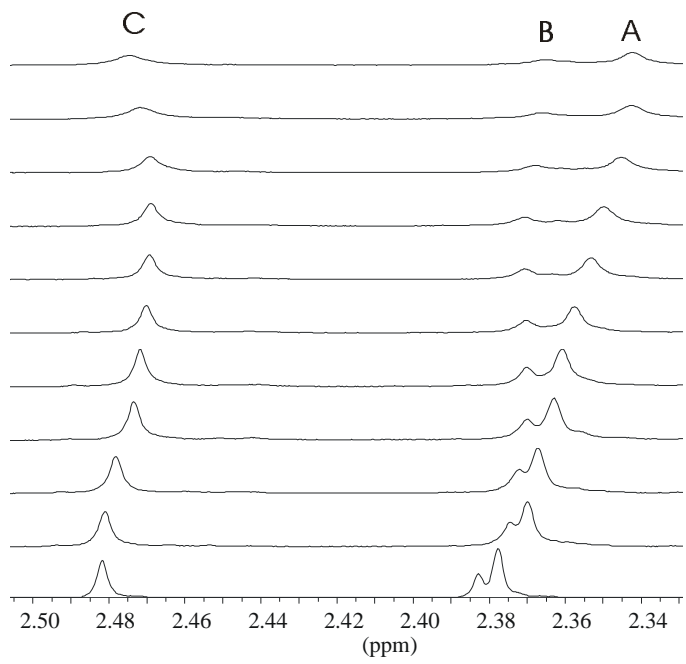

b)

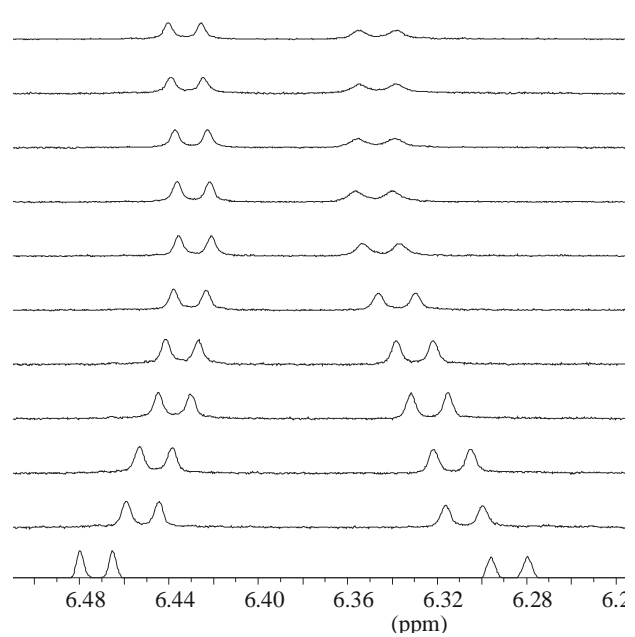

FIGURE S1. Partial ${ }^{1} \mathrm{H}$ NMR spectra $\left(500 \mathrm{MHz}, \mathrm{CDCl}_{3}, 25^{\circ} \mathrm{C}\right)$ of $\mathbf{1 1 b}$ after addition of (from bottom to top) $0,0.34,0.51,0.69,0.86,1.21,1.55,2.16,3.03,3.89$ and 4.32 equiv of $\mathbf{1 2 b}([\mathbf{1 1 b}]=$ $0.85 \mathrm{mM}$ ). (a) Chemical shifts of the $\mathrm{CH}_{3}$ resonances. (b) Chemical shifts of the pyridine $\mathrm{CH}$ resonances, HC-3 and HC-5.

\section{3. ${ }^{1} \mathrm{H}$ NMR Titration of $\mathbf{1 1 b}$ with $\alpha$-glucopyranoside 13 in $\mathrm{CDCl}_{3}$.}

a)

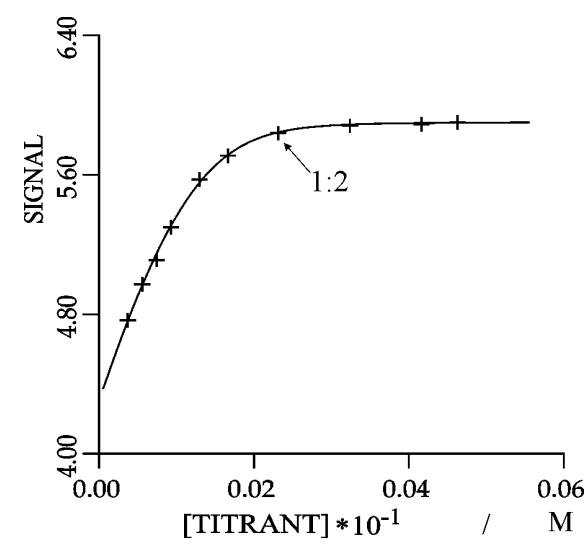

b)

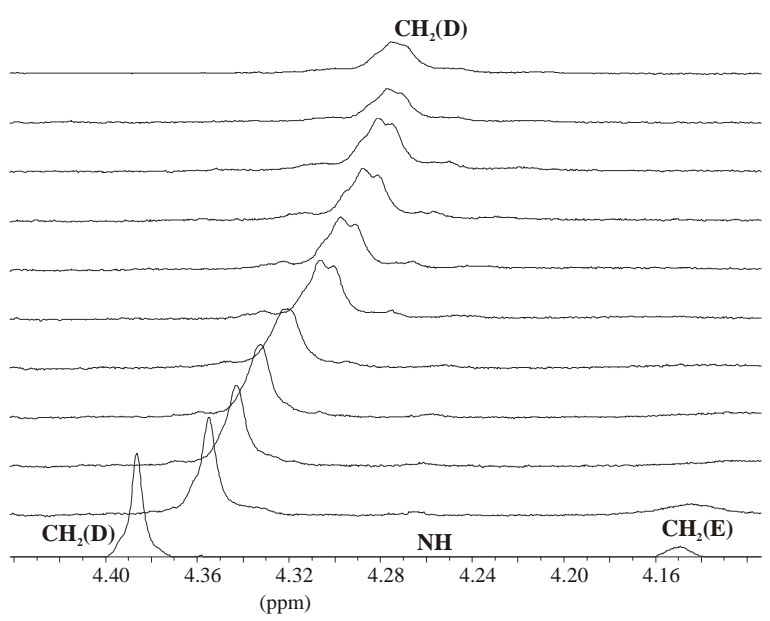

FIGURE S2. (a) Plot of the observed (x) and calculated (-) downfield chemical shifts of the NH resonances of $11 \mathrm{~b}$ as a function of added $\alpha$-glucopyranoside $13 ;[\mathbf{1 1 b}]=0.85 \mathrm{mM}$; Equiv of $13=$ $0.31,0.47,0.63,0.79,1.10,1.42,1.98,2.77,3.56$, and 3.96. The [receptor]:[sugar] ratio is marked. (b) Partial ${ }^{1} \mathrm{H}$ NMR spectra $\left(500 \mathrm{MHz}, \mathrm{CDCl}_{3}, 25^{\circ} \mathrm{C}\right)$ of $\mathbf{1 1 b}\left(\mathrm{CH}_{2}\right.$ resonances, D and E, are shown) after addition of (from bottom to top) $0,0.31,0.47,0.63,0.79,1.10,1.42,1.98,2.77,3.56$, and 3.96. equiv of 13. 
4. ${ }^{1} \mathrm{H}$ NMR Titration of receptor 9 with $\alpha$-glucopyranoside $\mathbf{1 3}$ in $\mathrm{CDCl}_{3}$.
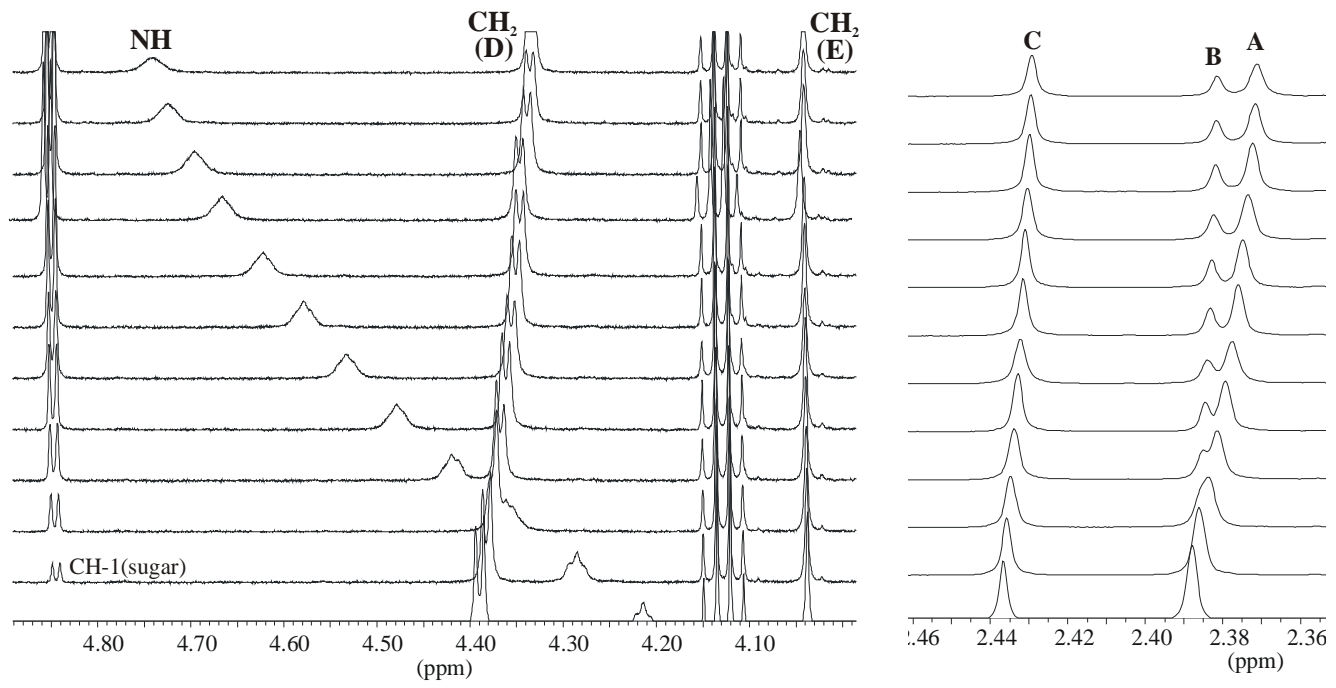

FIGURE S3. ${ }^{1} \mathrm{H}$ NMR titration of receptor 9 with $\alpha$-glucopyranoside 13 in $\mathrm{CDCl}_{3}$. Partial ${ }^{1} \mathrm{H}$ NMR spectra of 9 after addition of (from bottom to top) 0, 0.50, 1.01, 1.51, 2.02, 2.52, 3.03, 3.53, 4.04, 4.54, 5.05 and 6.00 equiv of $\mathbf{1 3}([\mathbf{9}]=0.91 \mathrm{mM})$.

5. ${ }^{1} \mathrm{H}$ NMR titration of receptor 11a with cellobiose $\mathbf{1 4}$ in $\mathrm{H}_{2} \mathrm{O} / \mathrm{D}_{2} \mathrm{O}$ (chemical shifts of the $\mathrm{CH}_{3}$ resonances)

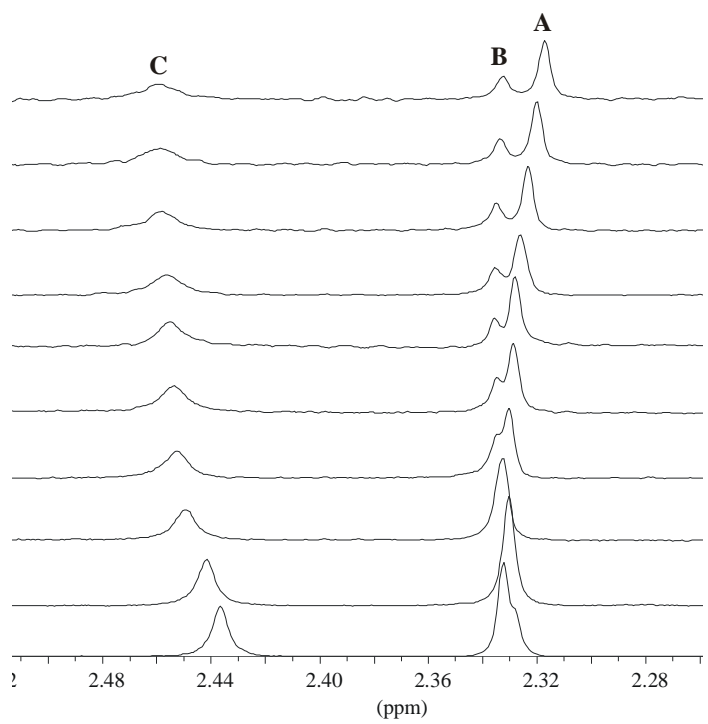

FIGURE S4. ) Partial ${ }^{1} \mathrm{H}$ NMR spectra of 11a in $\mathrm{H}_{2} \mathrm{O} / \mathrm{D}_{2} \mathrm{O}(93: 7 \mathrm{v} / \mathrm{v}$ ) after addition of (from bottom to top) $0,29,44,58,87,116,145,174,233,291$ and 349 equiv of $\mathbf{1 4}([\mathbf{1 1 a}]=0.72 \mathrm{mM})$. 
6. $\quad{ }^{1} \mathrm{H}$ and ${ }^{13} \mathrm{C}$ NMR spectra of compounds $9, \mathbf{1 0}, \mathbf{1 1} \mathbf{a}$ and $\mathbf{1 1 b}$.

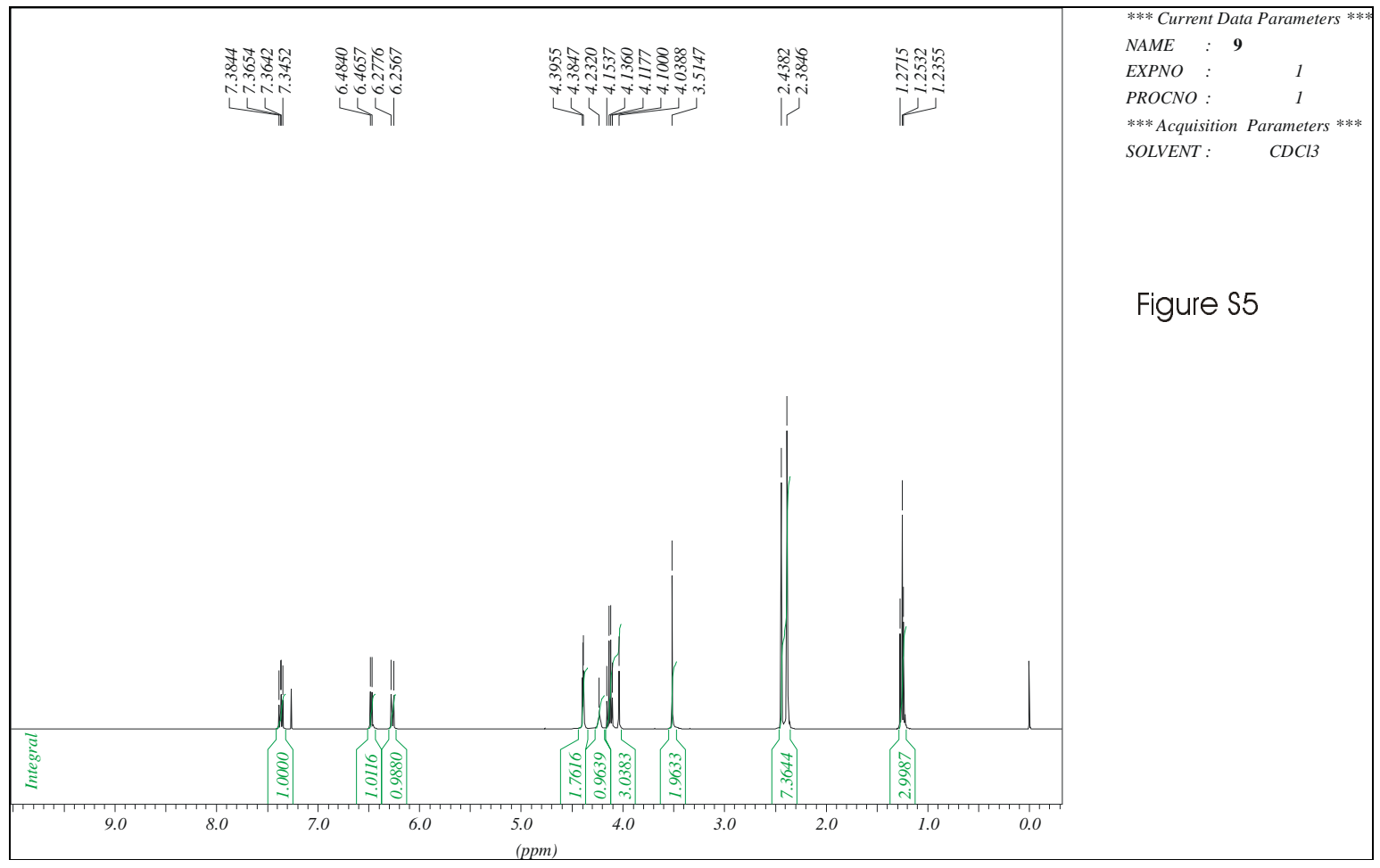

FIGURE S5. ${ }^{1} \mathrm{H}$ NMR spectrum of 9 in $\mathrm{CDCl}_{3}$.

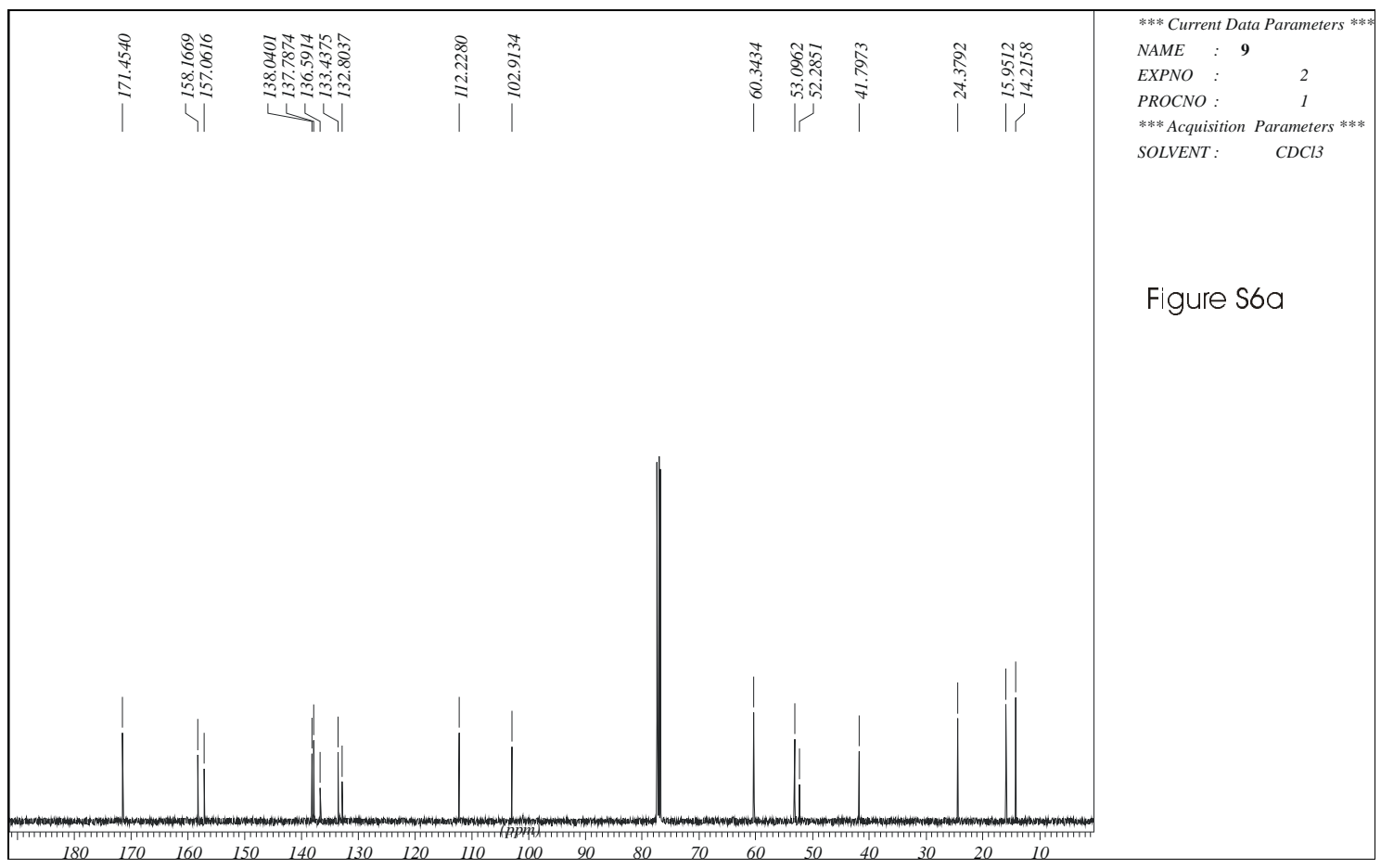




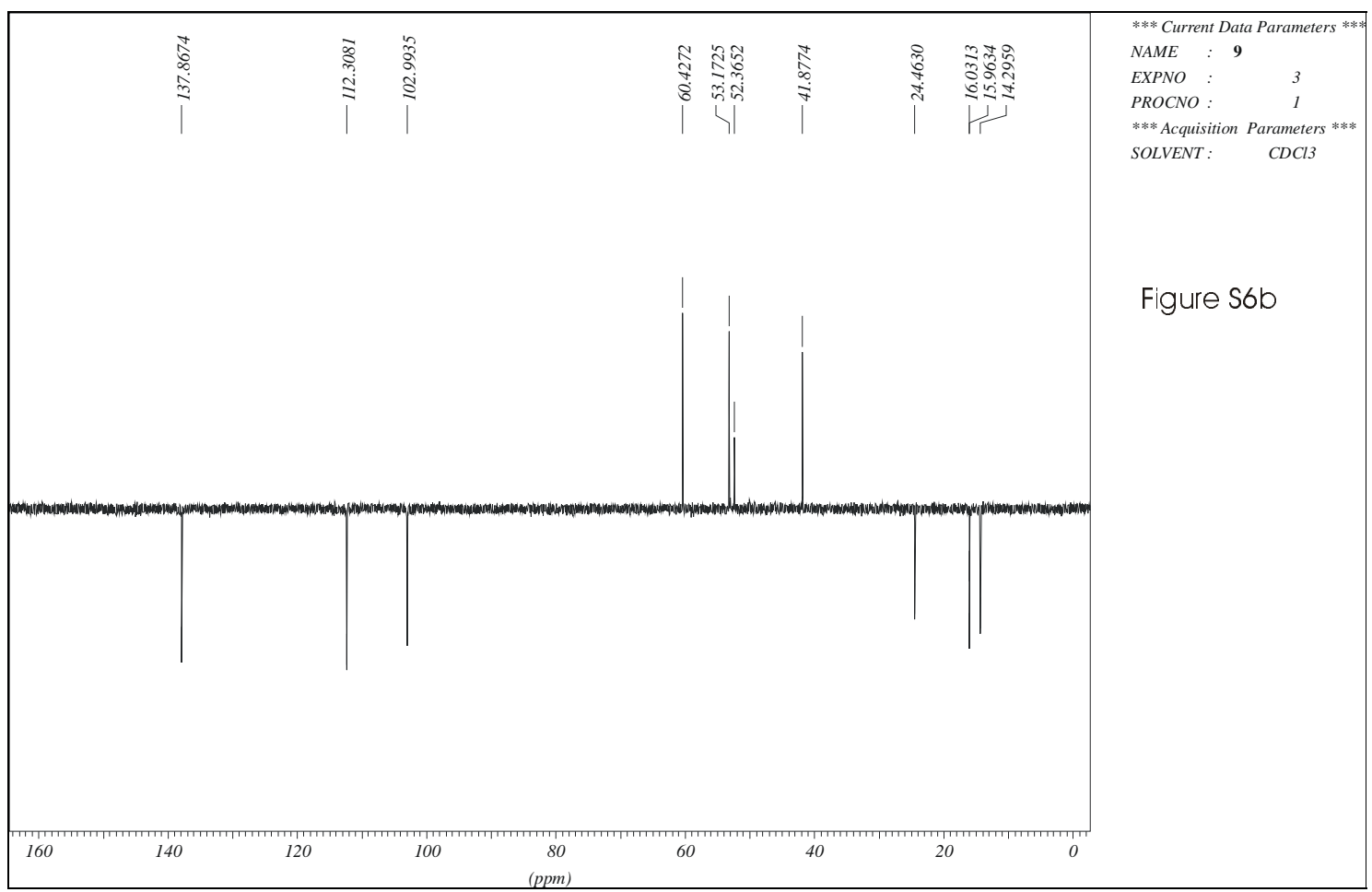

FIGURE S6. ${ }^{13} \mathrm{C}$ NMR spectra of 9 in $\mathrm{CDCl}_{3}$.

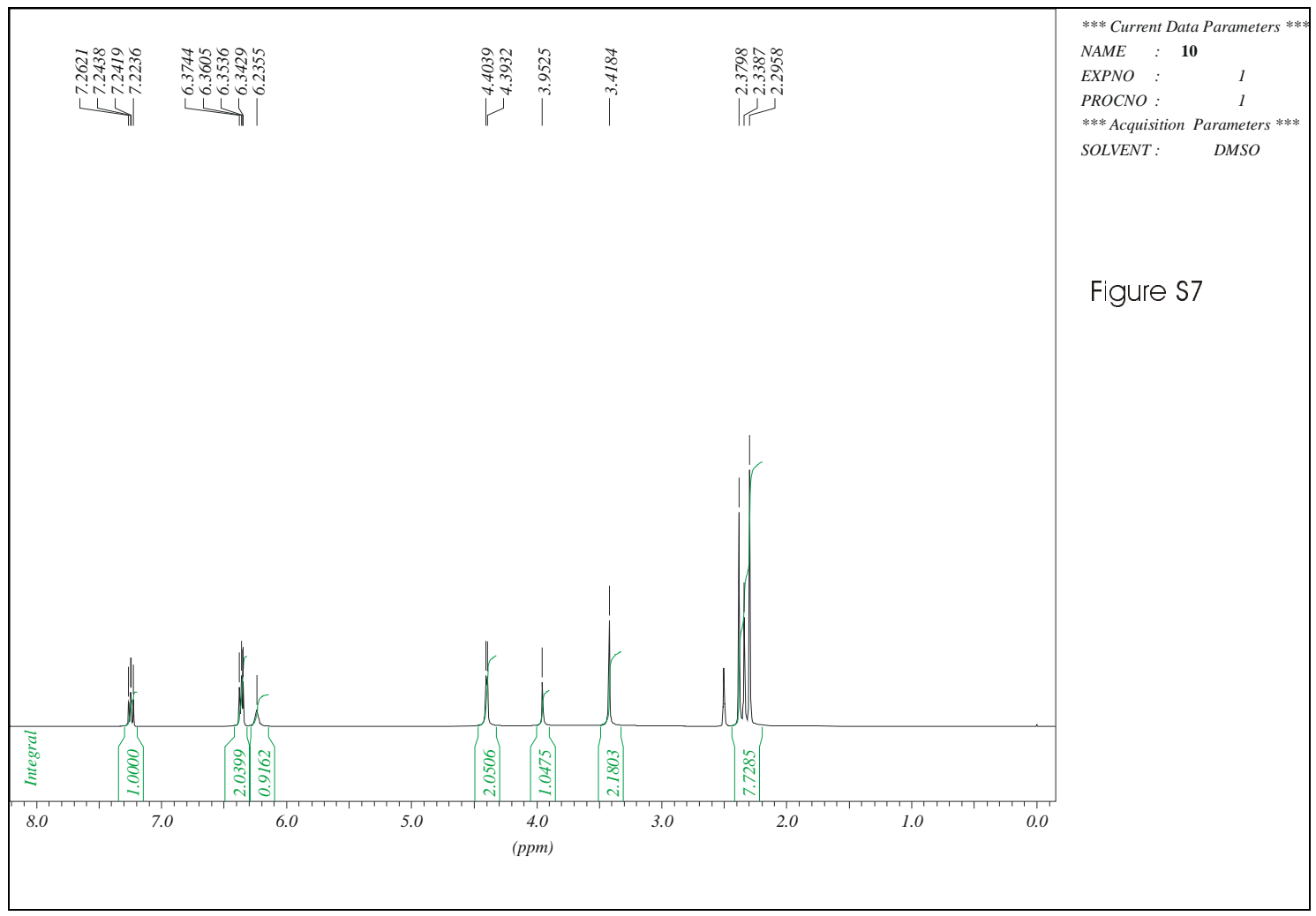

FIGURE S7. ${ }^{1} \mathrm{H}$ NMR spectrum of $\mathbf{1 0}$ in DMSO. 


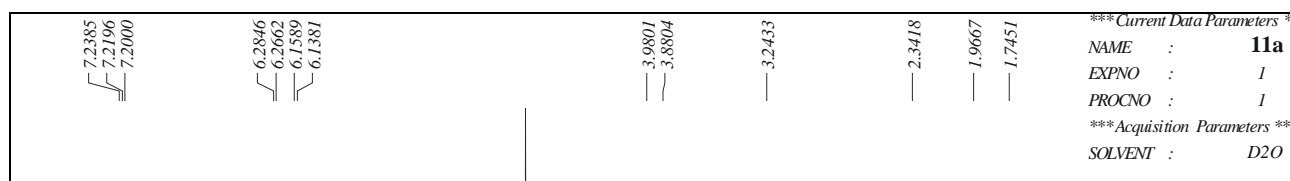

Figure S8a

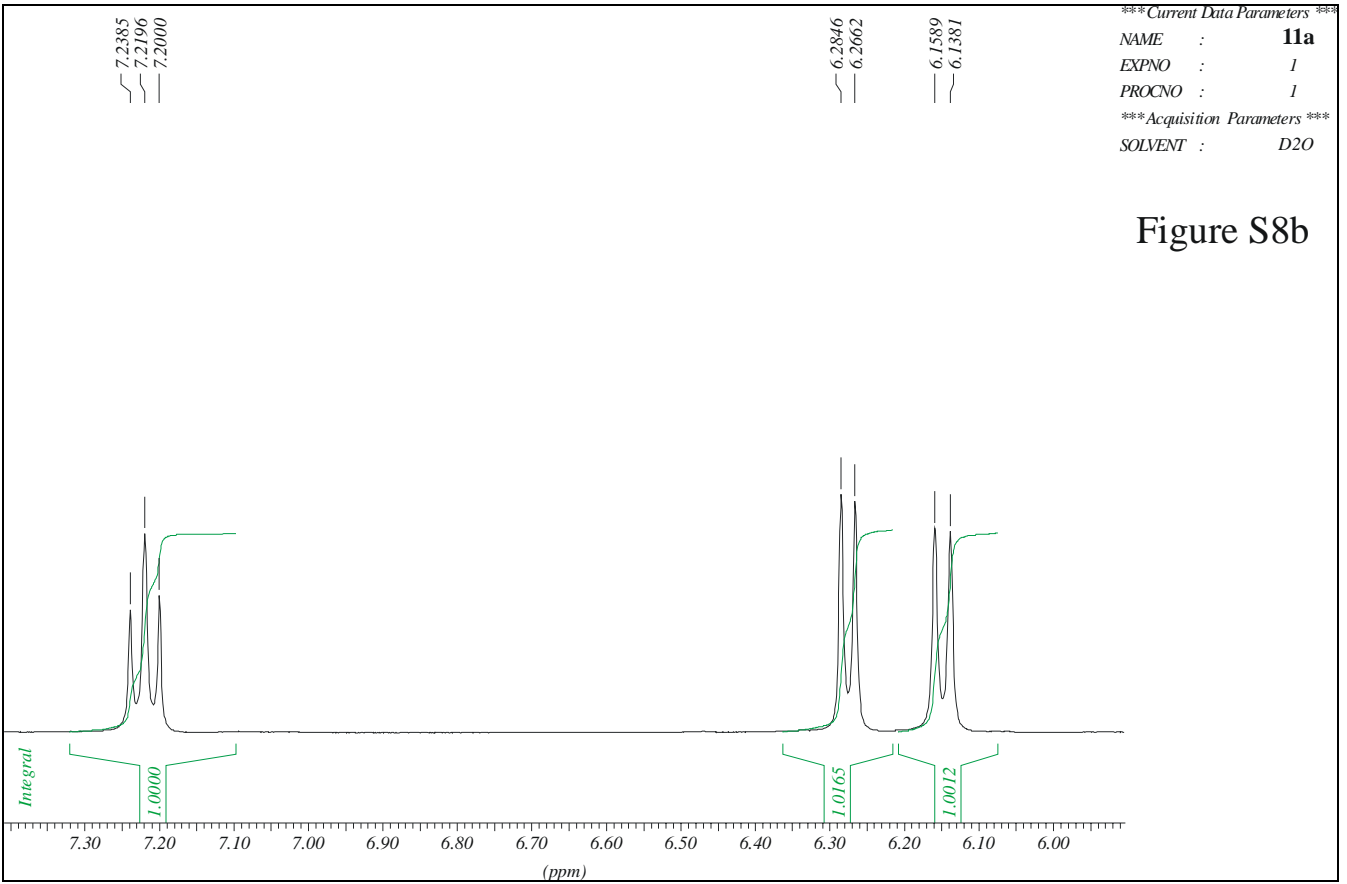

FIGURE S8. ${ }^{1} \mathrm{H}$ NMR spectra of 11a in $\mathrm{D}_{2} \mathrm{O}([11 \mathrm{a}]=45 \mathrm{mM})$. 

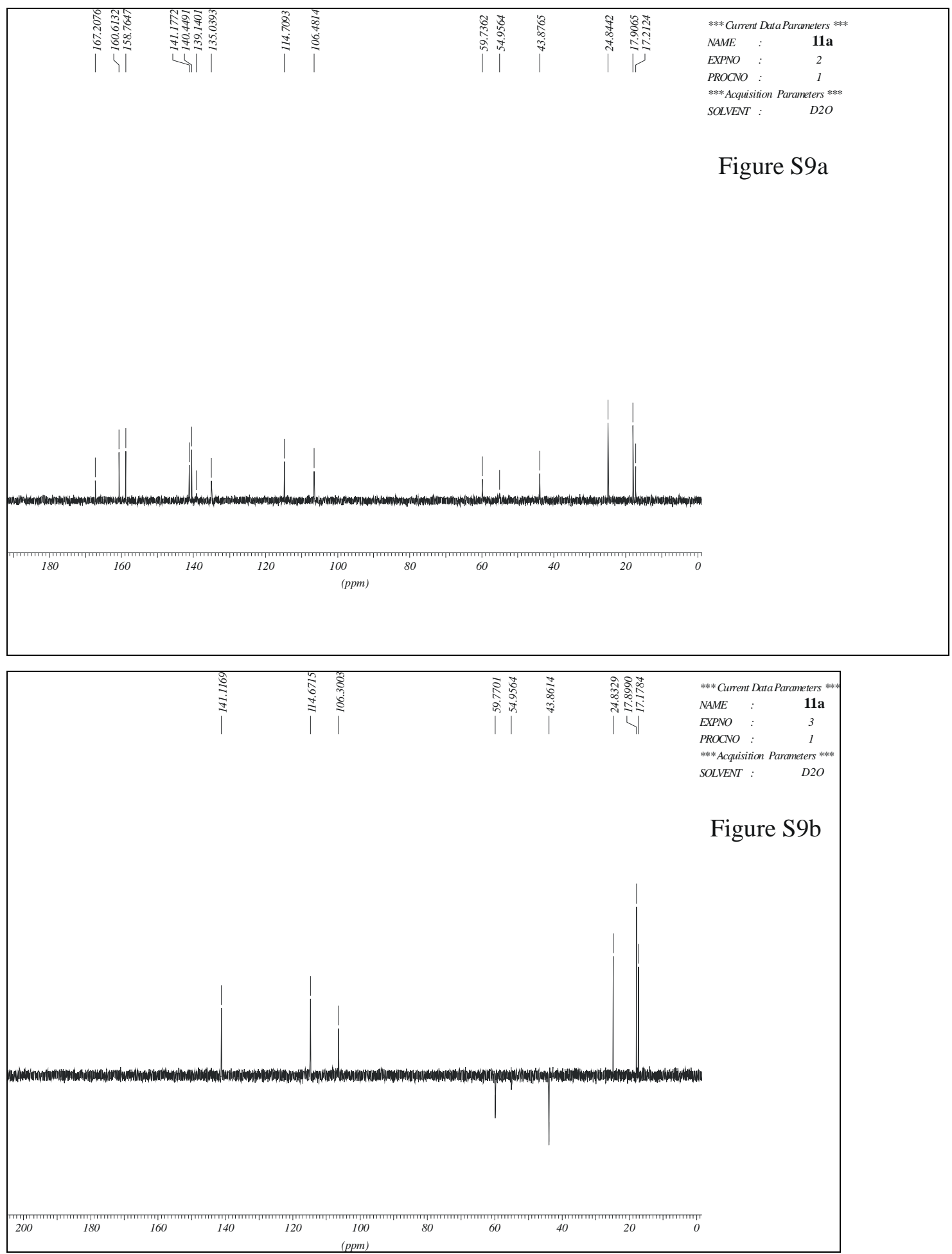

FIGURE S9. ${ }^{13} \mathrm{C}$ NMR spectra of 11a in $\mathrm{D}_{2} \mathrm{O}([\mathbf{1 1 a}]=45 \mathrm{mM})$. 


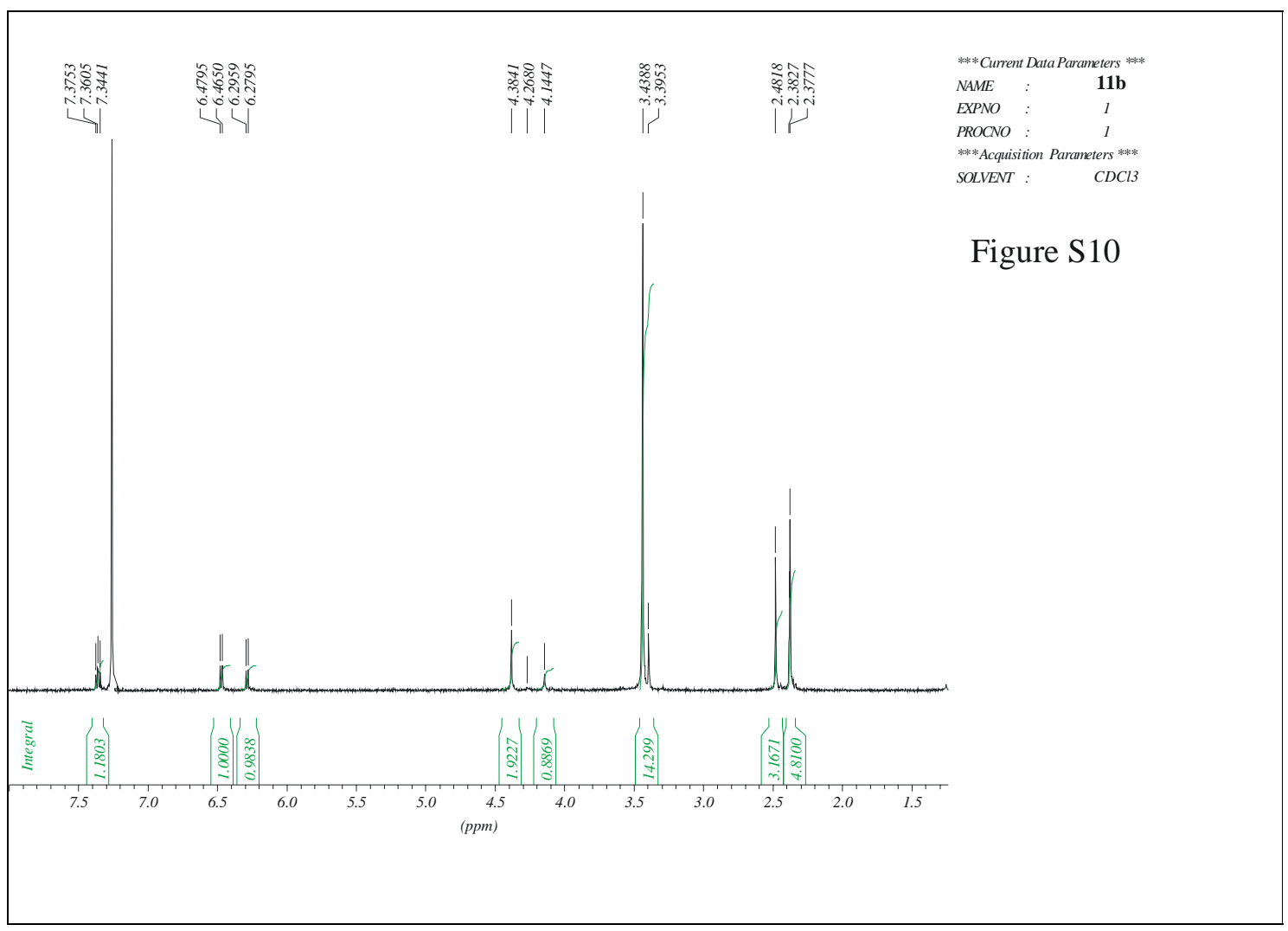

FIGURE S10. ${ }^{1} \mathrm{H}$ NMR spectrum of $11 \mathrm{a}$ in $\mathrm{CDCl}_{3}([\mathbf{1 1} \mathbf{b}]=0.85 \mathrm{mM})$.

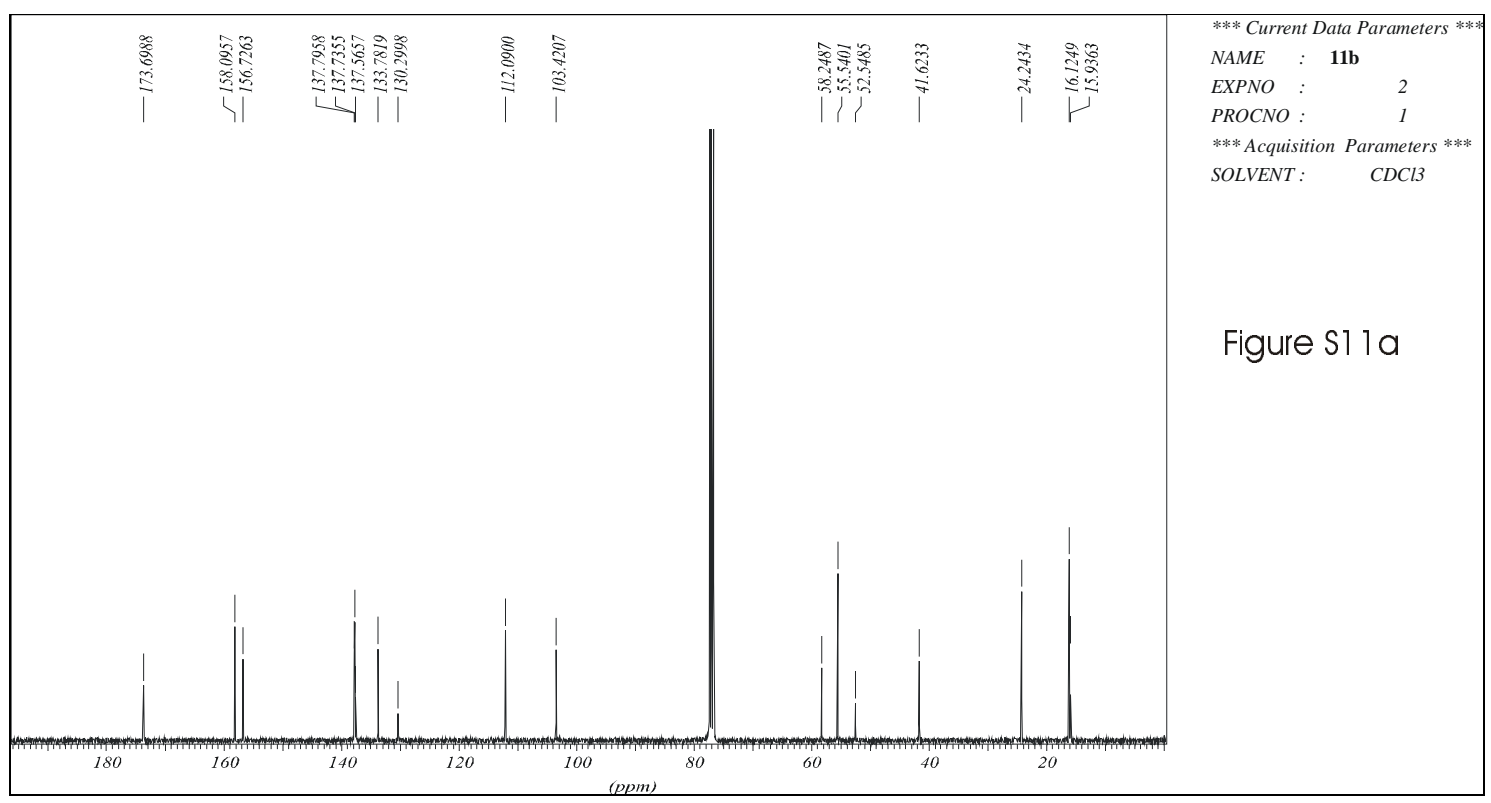




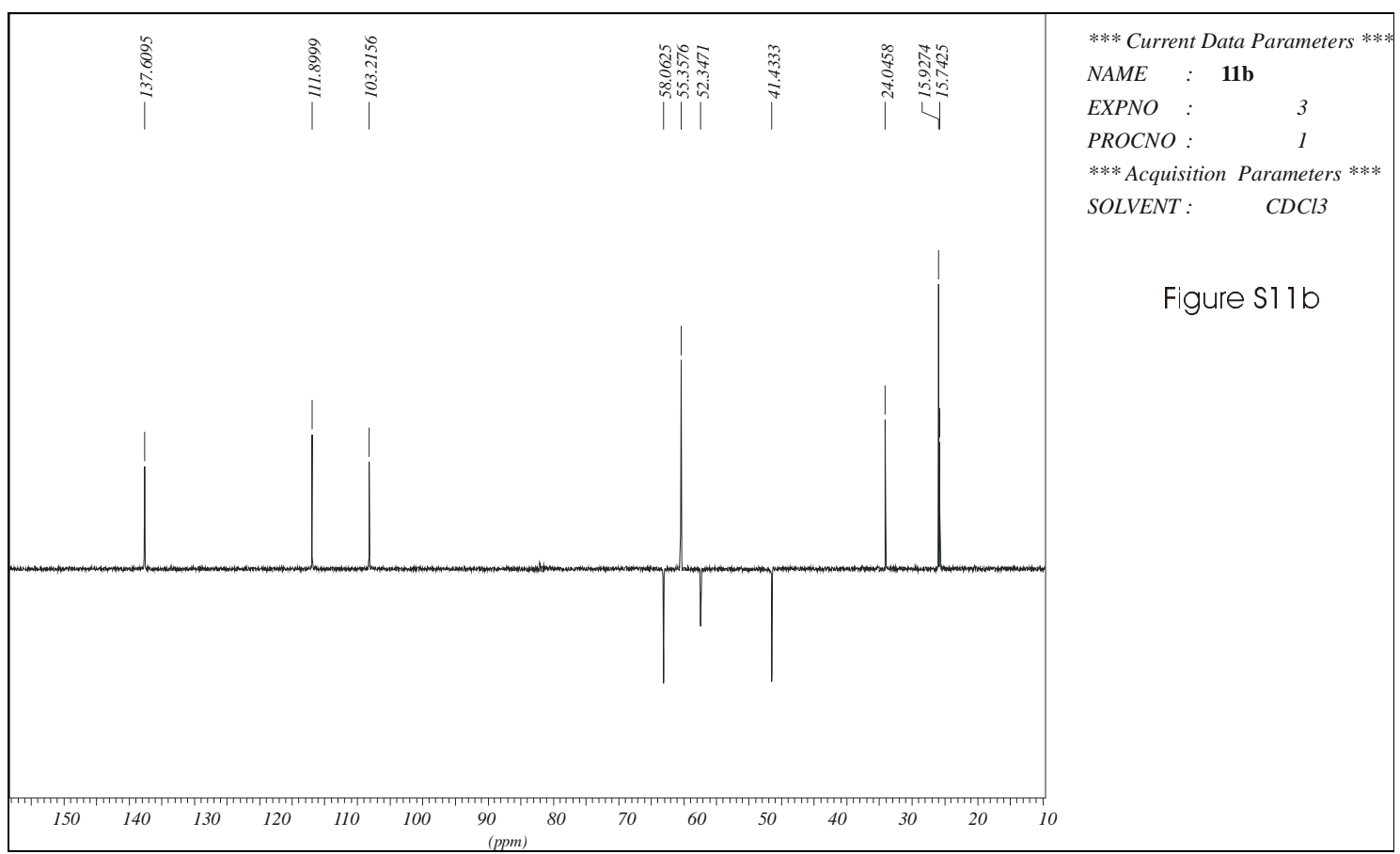

FIGURE S11. ${ }^{13} \mathrm{C}$ NMR spectra of $\mathbf{1 1 b}$ in $\mathrm{CDCl}_{3}([11 \mathbf{b}]=25 \mathrm{mM})$.

7. Crystal data for compound 9 (Tables of atomic coordinates and equivalent isotropic displacement parameters, bond lengths and angles, anisotropic displacement parameters, the anisotropic displacement factor, hydrogen coordinates and isotropic displacement parameters).

Table 1. Crystal data and structure refinement.

$\begin{array}{lll}\text { Identification code } & \text { majik } & \\ \text { Empirical formula } & \mathrm{C}_{32} \mathrm{H}_{43} \mathrm{~N}_{5} \mathrm{O}_{4} & \\ \text { Formula weight } & 561.71 & \\ \text { Temperature } & 133(2) \mathrm{K} & \\ \text { Wavelength } & 0.71073 \AA & \\ \text { Crystal system } & \text { Monoclinic } & \\ \text { Space group } & \mathrm{P} 2{ }_{1} / \mathrm{c} & \\ \text { Unit cell dimensions } & \mathrm{a}=11.6786(14) \AA & \square=90^{\circ} \\ & \mathrm{b}=24.579(3) \AA & \square=95.713(5)^{\circ} \\ \text { Volume } & \mathrm{c}=10.7047(12) \AA & \square=90^{\circ} \\ \text { Z } & 3057.5(6) \AA & \\ \text { Density (calculated) } & 4 & \\ \text { Absorption coefficient } & 1.220 \mathrm{Mg} / \mathrm{m}^{3} & \\ \mathrm{~F}(000) & 0.081 \mathrm{~mm}^{-1} & \\ \text { Crystal size } & 1208 & \\ \end{array}$


Theta range for data collection

Index ranges

Reflections collected

Independent reflections

Completeness to theta $=28.25^{\circ}$

Absorption correction

Refinement method

Data / restraints / parameters

Goodness-of-fit on $\mathrm{F}^{2}$

Final $\mathrm{R}$ indices [I>2sigma(I)]

$\mathrm{R}$ indices (all data)

Largest diff. peak and hole
1.66 to $28.27^{\circ}$

$-15<=\mathrm{h}<=15,-32<=\mathrm{k}<=32,-14<=\mathrm{l}<=14$

30954

$7583[\mathrm{R}($ int $)=0.0732]$

$99.8 \%$

None

Full-matrix least-squares on $\mathrm{F}^{2}$

7583 / 0 / 385

0.938

$\mathrm{R} 1=0.0525, \mathrm{wR} 2=0.1222$

$\mathrm{R} 1=0.0998, \mathrm{wR} 2=0.1385$

0.278 and -0.291 e. $\AA^{-3}$

Table 2. Atomic coordinates ( $\mathrm{x} 10^{4}$ ) and equivalent isotropic displacement parameters $\left(\AA^{2} \mathrm{X} 10^{3}\right)$. U(eq) is defined as one third of the trace of the orthogonalized $\mathrm{U}_{\mathrm{ij}}$ tensor.

\begin{tabular}{lcccc}
\hline & $\mathrm{x}$ & $\mathrm{y}$ & $\mathrm{z}$ & $\mathrm{U}(\mathrm{eq})$ \\
\hline $\mathrm{C}(1)$ & & & & \\
$\mathrm{C}(2)$ & $4689.6(14)$ & $5363.6(7)$ & $3209.4(15)$ & $20.5(4)$ \\
$\mathrm{C}(3)$ & $4841.1(15)$ & $4874.0(7)$ & $2571.9(16)$ & $22.2(4)$ \\
$\mathrm{C}(4)$ & $5650.9(15)$ & $4848.6(7)$ & $1682.4(16)$ & $22.4(4)$ \\
$\mathrm{C}(5)$ & $6327.6(15)$ & $5301.5(7)$ & $1464.5(15)$ & $21.3(4)$ \\
$\mathrm{C}(6)$ & $6155.4(14)$ & $5793.8(7)$ & $2086.6(15)$ & $19.6(4)$ \\
$\mathrm{C}(7)$ & $5331.1(15)$ & $5829.7(7)$ & $2946.4(15)$ & $19.5(4)$ \\
$\mathrm{C}(8)$ & $5128.5(16)$ & $6359.8(7)$ & $3611.2(18)$ & $28.3(4)$ \\
$\mathrm{C}(9)$ & $4148.2(17)$ & $4366.6(8)$ & $2815.2(19)$ & $33.3(5)$ \\
$\mathrm{C}(10)$ & $7264.3(17)$ & $5261.8(8)$ & $588.8(17)$ & $31.9(5)$ \\
$\mathrm{C}(11)$ & $6854.6(15)$ & $6289.0(7)$ & $1785.5(16)$ & $23.8(4)$ \\
$\mathrm{C}(12)$ & $3859.4(15)$ & $5406.1(8)$ & $4206.4(16)$ & $25.3(4)$ \\
$\mathrm{C}(13)$ & $5795.8(17)$ & $4319.4(7)$ & $979.4(18)$ & $29.3(4)$ \\
$\mathrm{C}(14)$ & $6859.6(15)$ & $3449.3(7)$ & $1368.2(16)$ & $21.7(4)$ \\
$\mathrm{C}(15)$ & $7773.5(16)$ & $3165.5(8)$ & $2030.2(17)$ & $27.1(4)$ \\
$\mathrm{C}(16)$ & $7931.2(17)$ & $2631.2(8)$ & $1745.5(18)$ & $30.5(5)$ \\
$\mathrm{C}(17)$ & $7214.5(16)$ & $2389.9(7)$ & $792.7(18)$ & $29.2(4)$ \\
$\mathrm{C}(18)$ & $6356.2(15)$ & $2693.1(7)$ & $157.4(17)$ & $24.5(4)$ \\
$\mathrm{C}(19)$ & $5573.9(17)$ & $2451.7(8)$ & $-901(2)$ & $35.6(5)$ \\
$\mathrm{C}(20)$ & $7059.1(15)$ & $6854.6(7)$ & $-93.2(16)$ & $20.0(4)$ \\
$\mathrm{C}(21)$ & $6638.7(16)$ & $7014.3(7)$ & $-1323.4(16)$ & $25.1(4)$ \\
$\mathrm{C}(22)$ & $7322.0(16)$ & $7331.6(7)$ & $-1980.3(17)$ & $28.0(4)$ \\
$\mathrm{C}(23)$ & $8395.6(16)$ & $7501.9(7)$ & $-1425.5(17)$ & $26.2(4)$ \\
& $8735.6(15)$ & $7342.5(7)$ & $-205.6(16)$ & $22.1(4)$ \\
& & & &
\end{tabular}




$\begin{array}{lrrrr}\mathrm{C}(24) & 9840.5(16) & 7536.3(8) & 496.4(18) & 31.0(4) \\ \mathrm{C}(25) & 2156.9(15) & 5493.7(7) & 2653.1(16) & 23.8(4) \\ \mathrm{C}(26) & 2133.0(16) & 5833.3(7) & 4817.6(17) & 24.5(4) \\ \mathrm{C}(27) & 1394.6(16) & 5917.3(7) & 1974.0(17) & 24.6(4) \\ \mathrm{C}(28) & 1718.5(15) & 5372.6(7) & 5605.7(17) & 24.0(4) \\ \mathrm{C}(29) & 211.2(16) & 6086.9(8) & 79.0(18) & 30.8(5) \\ \mathrm{C}(30) & 1244.1(18) & 4438.3(8) & 5736.0(19) & 35.9(5) \\ \mathrm{C}(31) & -283.0(18) & 5771.4(9) & -1048.5(18) & 38.6(5) \\ \mathrm{C}(32) & 1233(2) & 3943.3(9) & 4924(3) & 57.2(7) \\ \mathrm{N}(1) & 6419.3(14) & 6508.9(6) & 571.1(14) & 24.7(4) \\ \mathrm{N}(2) & 2799.7(12) & 5703.3(6) & 3783.1(13) & 22.1(3) \\ \mathrm{N}(3) & 6639.4(14) & 3976.9(6) & 1700.9(15) & 29.2(4) \\ \mathrm{N}(4) & 6163.5(12) & 3222.4(6) & 440.4(14) & 23.0(3) \\ \mathrm{N}(5) & 8085.1(12) & 7013.4(6) & 453.0(13) & 20.7(3) \\ \mathrm{O}(1) & 1148.7(13) & 6354.4(5) & 2375.5(13) & 40.9(4) \\ \mathrm{O}(2) & 1488.9(12) & 5432.8(6) & 6669.2(12) & 36.6(4) \\ \mathrm{O}(3) & 989.9(11) & 5727.1(5) & 840.3(11) & 28.2(3) \\ \mathrm{O}(4) & 1606.7(11) & 4895.4(5) & 4999.9(12) & 29.5(3) \\ & & & & \end{array}$

Table 3. Bond lengths $[\AA]$ and angles $\left[^{\circ}\right]$.

\begin{tabular}{llll}
\hline $\mathrm{C}(1)-\mathrm{C}(2)$ & $1.403(2)$ & $\mathrm{C}(13)-\mathrm{C}(14)$ & $1.406(2)$ \\
$\mathrm{C}(1)-\mathrm{C}(6)$ & $1.412(2)$ & $\mathrm{C}(14)-\mathrm{C}(15)$ & $1.365(3)$ \\
$\mathrm{C}(1)-\mathrm{C}(11)$ & $1.515(2)$ & $\mathrm{C}(15)-\mathrm{C}(16)$ & $1.387(3)$ \\
$\mathrm{C}(2)-\mathrm{C}(3)$ & $1.409(3)$ & $\mathrm{C}(16)-\mathrm{C}(17)$ & $1.374(2)$ \\
$\mathrm{C}(2)-\mathrm{C}(8)$ & $1.523(2)$ & $\mathrm{C}(17)-\mathrm{N}(4)$ & $1.359(2)$ \\
$\mathrm{C}(3)-\mathrm{C}(4)$ & $1.398(2)$ & $\mathrm{C}(17)-\mathrm{C}(18)$ & $1.505(3)$ \\
$\mathrm{C}(3)-\mathrm{C}(12)$ & $1.520(2)$ & $\mathrm{C}(19)-\mathrm{N}(5)$ & $1.338(2)$ \\
$\mathrm{C}(4)-\mathrm{C}(5)$ & $1.405(2)$ & $\mathrm{C}(19)-\mathrm{N}(1)$ & $1.375(2)$ \\
$\mathrm{C}(4)-\mathrm{C}(9)$ & $1.513(3)$ & $\mathrm{C}(19)-\mathrm{C}(20)$ & $1.414(2)$ \\
$\mathrm{C}(5)-\mathrm{C}(6)$ & $1.399(2)$ & $\mathrm{C}(20)-\mathrm{C}(21)$ & $1.361(3)$ \\
$\mathrm{C}(5)-\mathrm{C}(10)$ & $1.518(2)$ & $\mathrm{C}(21)-\mathrm{C}(22)$ & $1.397(3)$ \\
$\mathrm{C}(6)-\mathrm{C}(7)$ & $1.515(2)$ & $\mathrm{C}(22)-\mathrm{C}(23)$ & $1.383(2)$ \\
$\mathrm{C}(10)-\mathrm{N}(1)$ & $1.452(2)$ & $\mathrm{C}(23)-\mathrm{N}(5)$ & $1.355(2)$ \\
$\mathrm{C}(11)-\mathrm{N}(2)$ & $1.469(2)$ & $\mathrm{C}(23)-\mathrm{C}(24)$ & $1.504(2)$ \\
$\mathrm{C}(12)-\mathrm{N}(3)$ & $1.458(2)$ & $\mathrm{C}(25)-\mathrm{N}(2)$ & $1.453(2)$ \\
$\mathrm{C}(13)-\mathrm{N}(4)$ & $1.341(2)$ & $\mathrm{C}(25)-\mathrm{C}(27)$ & $1.509(2)$ \\
$\mathrm{C}(13)-\mathrm{N}(3)$ & $1.376(2)$ & $\mathrm{C}(26)-\mathrm{N}(2)$ & $1.451(2)$
\end{tabular}




\begin{tabular}{|c|c|c|c|}
\hline $\mathrm{C}(26)-\mathrm{C}(28)$ & $1.520(2)$ & $\mathrm{C}(29)-\mathrm{O}(3)$ & $1.458(2)$ \\
\hline $\mathrm{C}(27)-\mathrm{O}(1)$ & $1.203(2)$ & $\mathrm{C}(29)-\mathrm{C}(31)$ & $1.501(3)$ \\
\hline $\mathrm{C}(27)-\mathrm{O}(3)$ & $1.342(2)$ & $\mathrm{C}(30)-\mathrm{O}(4)$ & $1.459(2)$ \\
\hline $\mathrm{C}(28)-\mathrm{O}(2)$ & $1.204(2)$ & $C(30)-C(32)$ & $1.495(3)$ \\
\hline $\mathrm{C}(28)-\mathrm{O}(4)$ & $1.340(2)$ & & \\
\hline $\mathrm{C}(2)-\mathrm{C}(1)-\mathrm{C}(6)$ & $120.23(15)$ & $C(16)-C(17)-C(18)$ & $121.15(16)$ \\
\hline $\mathrm{C}(2)-\mathrm{C}(1)-\mathrm{C}(11)$ & $121.48(15)$ & $\mathrm{N}(5)-\mathrm{C}(19)-\mathrm{N}(1)$ & $117.60(15)$ \\
\hline$C(6)-C(1)-C(11)$ & $118.28(15)$ & $\mathrm{N}(5)-\mathrm{C}(19)-\mathrm{C}(20)$ & $122.58(16)$ \\
\hline $\mathrm{C}(1)-\mathrm{C}(2)-\mathrm{C}(3)$ & $119.50(15)$ & $\mathrm{N}(1)-\mathrm{C}(19)-\mathrm{C}(20)$ & $119.79(16)$ \\
\hline $\mathrm{C}(1)-\mathrm{C}(2)-\mathrm{C}(8)$ & 121.61(16) & $\mathrm{C}(21)-\mathrm{C}(20)-\mathrm{C}(19)$ & $118.24(17)$ \\
\hline $\mathrm{C}(3)-\mathrm{C}(2)-\mathrm{C}(8)$ & $118.89(16)$ & $C(20)-C(21)-C(22)$ & $119.98(17)$ \\
\hline $\mathrm{C}(4)-\mathrm{C}(3)-\mathrm{C}(2)$ & $120.41(16)$ & $\mathrm{C}(23)-\mathrm{C}(22)-\mathrm{C}(21)$ & $118.54(17)$ \\
\hline $\mathrm{C}(4)-\mathrm{C}(3)-\mathrm{C}(12)$ & $120.39(16)$ & $\mathrm{N}(5)-\mathrm{C}(23)-\mathrm{C}(22)$ & $122.49(16)$ \\
\hline $\mathrm{C}(2)-\mathrm{C}(3)-\mathrm{C}(12)$ & $119.20(16)$ & $\mathrm{N}(5)-\mathrm{C}(23)-\mathrm{C}(24)$ & $115.26(15)$ \\
\hline $\mathrm{C}(3)-\mathrm{C}(4)-\mathrm{C}(5)$ & 119.77(16) & $\mathrm{C}(22)-\mathrm{C}(23)-\mathrm{C}(24)$ & $122.22(16)$ \\
\hline $\mathrm{C}(3)-\mathrm{C}(4)-\mathrm{C}(9)$ & $120.51(16)$ & $\mathrm{N}(2)-\mathrm{C}(25)-\mathrm{C}(27)$ & $112.81(14)$ \\
\hline$C(5)-C(4)-C(9)$ & $119.70(16)$ & $\mathrm{N}(2)-\mathrm{C}(26)-\mathrm{C}(28)$ & $118.96(15)$ \\
\hline$C(6)-C(5)-C(4)$ & $120.42(16)$ & $\mathrm{O}(1)-\mathrm{C}(27)-\mathrm{O}(3)$ & $124.01(17)$ \\
\hline$C(6)-C(5)-C(10)$ & $120.59(15)$ & $\mathrm{O}(1)-\mathrm{C}(27)-\mathrm{C}(25)$ & $126.50(17)$ \\
\hline $\mathrm{C}(4)-\mathrm{C}(5)-\mathrm{C}(10)$ & $118.97(15)$ & $\mathrm{O}(3)-\mathrm{C}(27)-\mathrm{C}(25)$ & $109.47(15)$ \\
\hline$C(5)-C(6)-C(1)$ & $119.55(15)$ & $\mathrm{O}(2)-\mathrm{C}(28)-\mathrm{O}(4)$ & $123.01(17)$ \\
\hline$C(5)-C(6)-C(7)$ & $120.88(15)$ & $\mathrm{O}(2)-\mathrm{C}(28)-\mathrm{C}(26)$ & $123.11(17)$ \\
\hline$C(1)-C(6)-C(7)$ & $119.56(15)$ & $\mathrm{O}(4)-\mathrm{C}(28)-\mathrm{C}(26)$ & $113.86(15)$ \\
\hline $\mathrm{N}(1)-\mathrm{C}(10)-\mathrm{C}(5)$ & 109.93(14) & $\mathrm{O}(3)-\mathrm{C}(29)-\mathrm{C}(31)$ & $107.73(16)$ \\
\hline $\mathrm{N}(2)-\mathrm{C}(11)-\mathrm{C}(1)$ & $113.29(14)$ & $\mathrm{O}(4)-\mathrm{C}(30)-\mathrm{C}(32)$ & $107.40(17)$ \\
\hline $\mathrm{N}(3)-\mathrm{C}(12)-\mathrm{C}(3)$ & $109.54(14)$ & $\mathrm{C}(19)-\mathrm{N}(1)-\mathrm{C}(10)$ & $122.21(15)$ \\
\hline $\mathrm{N}(4)-\mathrm{C}(13)-\mathrm{N}(3)$ & $117.95(15)$ & $\mathrm{C}(26)-\mathrm{N}(2)-\mathrm{C}(25)$ & $116.18(14)$ \\
\hline $\mathrm{N}(4)-\mathrm{C}(13)-\mathrm{C}(14)$ & $122.73(16)$ & $\mathrm{C}(26)-\mathrm{N}(2)-\mathrm{C}(11)$ & $111.93(14)$ \\
\hline $\mathrm{N}(3)-\mathrm{C}(13)-\mathrm{C}(14)$ & 119.29(16) & $\mathrm{C}(25)-\mathrm{N}(2)-\mathrm{C}(11)$ & $115.46(14)$ \\
\hline $\mathrm{C}(15)-\mathrm{C}(14)-\mathrm{C}(13)$ & $118.52(17)$ & $\mathrm{C}(13)-\mathrm{N}(3)-\mathrm{C}(12)$ & $122.76(15)$ \\
\hline$C(14)-C(15)-C(16)$ & $119.40(17)$ & $\mathrm{C}(13)-\mathrm{N}(4)-\mathrm{C}(17)$ & $117.49(15)$ \\
\hline$C(17)-C(16)-C(15)$ & 119.29(17) & $\mathrm{C}(19)-\mathrm{N}(5)-\mathrm{C}(23)$ & $118.10(15)$ \\
\hline $\mathrm{N}(4)-\mathrm{C}(17)-\mathrm{C}(16)$ & $122.53(16)$ & $\mathrm{C}(27)-\mathrm{O}(3)-\mathrm{C}(29)$ & $115.99(14)$ \\
\hline $\mathrm{N}(4)-\mathrm{C}(17)-\mathrm{C}(18)$ & 116.32(16) & $\mathrm{C}(28)-\mathrm{O}(4)-\mathrm{C}(30)$ & $115.60(14)$ \\
\hline
\end{tabular}


Table 4. Torsion angles $\left[{ }^{\circ}\right]$.

\begin{tabular}{|c|c|c|c|}
\hline $\mathrm{C}(6)-\mathrm{C}(1)-\mathrm{C}(2)-\mathrm{C}(3)$ & $1.0(2)$ & $\mathrm{N}(1)-\mathrm{C}(19)-\mathrm{C}(20)-\mathrm{C}(21)$ & $-176.33(16)$ \\
\hline $\mathrm{C}(11)-\mathrm{C}(1)-\mathrm{C}(2)-\mathrm{C}(3)$ & $-177.86(15)$ & $C(19)-C(20)-C(21)-C(22)$ & $-1.6(3)$ \\
\hline $\mathrm{C}(6)-\mathrm{C}(1)-\mathrm{C}(2)-\mathrm{C}(8)$ & $-178.88(15)$ & $C(20)-C(21)-C(22)-C(23)$ & $-0.2(3)$ \\
\hline $\mathrm{C}(11)-\mathrm{C}(1)-\mathrm{C}(2)-\mathrm{C}(8)$ & $2.3(2)$ & $\mathrm{C}(21)-\mathrm{C}(22)-\mathrm{C}(23)-\mathrm{N}(5)$ & $2.3(3)$ \\
\hline $\mathrm{C}(1)-\mathrm{C}(2)-\mathrm{C}(3)-\mathrm{C}(4)$ & $2.1(2)$ & $\mathrm{C}(21)-\mathrm{C}(22)-\mathrm{C}(23)-\mathrm{C}(24)$ & $-175.89(17)$ \\
\hline $\mathrm{C}(8)-\mathrm{C}(2)-\mathrm{C}(3)-\mathrm{C}(4)$ & $-177.96(16)$ & $\mathrm{N}(2)-\mathrm{C}(25)-\mathrm{C}(27)-\mathrm{O}(1)$ & $-12.7(3)$ \\
\hline $\mathrm{C}(1)-\mathrm{C}(2)-\mathrm{C}(3)-\mathrm{C}(12)$ & $-178.97(15)$ & $\mathrm{N}(2)-\mathrm{C}(25)-\mathrm{C}(27)-\mathrm{O}(3)$ & $169.12(14)$ \\
\hline $\mathrm{C}(8)-\mathrm{C}(2)-\mathrm{C}(3)-\mathrm{C}(12)$ & $0.9(2)$ & $\mathrm{N}(2)-\mathrm{C}(26)-\mathrm{C}(28)-\mathrm{O}(2)$ & $156.22(17)$ \\
\hline $\mathrm{C}(2)-\mathrm{C}(3)-\mathrm{C}(4)-\mathrm{C}(5)$ & $-3.5(2)$ & $\mathrm{N}(2)-\mathrm{C}(26)-\mathrm{C}(28)-\mathrm{O}(4)$ & $-25.2(2)$ \\
\hline $\mathrm{C}(12)-\mathrm{C}(3)-\mathrm{C}(4)-\mathrm{C}(5)$ & $177.67(15)$ & $\mathrm{N}(5)-\mathrm{C}(19)-\mathrm{N}(1)-\mathrm{C}(10)$ & $-4.2(2)$ \\
\hline $\mathrm{C}(2)-\mathrm{C}(3)-\mathrm{C}(4)-\mathrm{C}(9)$ & $175.00(16)$ & $\mathrm{C}(20)-\mathrm{C}(19)-\mathrm{N}(1)-\mathrm{C}(10)$ & $173.83(16)$ \\
\hline $\mathrm{C}(12)-\mathrm{C}(3)-\mathrm{C}(4)-\mathrm{C}(9)$ & $-3.9(2)$ & $\mathrm{C}(5)-\mathrm{C}(10)-\mathrm{N}(1)-\mathrm{C}(19)$ & $-161.61(16)$ \\
\hline $\mathrm{C}(3)-\mathrm{C}(4)-\mathrm{C}(5)-\mathrm{C}(6)$ & $1.6(2)$ & $\mathrm{C}(28)-\mathrm{C}(26)-\mathrm{N}(2)-\mathrm{C}(25)$ & $76.6(2)$ \\
\hline $\mathrm{C}(9)-\mathrm{C}(4)-\mathrm{C}(5)-\mathrm{C}(6)$ & $-176.85(15)$ & $\mathrm{C}(28)-\mathrm{C}(26)-\mathrm{N}(2)-\mathrm{C}(11)$ & $-59.0(2)$ \\
\hline $\mathrm{C}(3)-\mathrm{C}(4)-\mathrm{C}(5)-\mathrm{C}(10)$ & $-176.77(15)$ & $\mathrm{C}(27)-\mathrm{C}(25)-\mathrm{N}(2)-\mathrm{C}(26)$ & $69.4(2)$ \\
\hline $\mathrm{C}(9)-\mathrm{C}(4)-\mathrm{C}(5)-\mathrm{C}(10)$ & $4.8(2)$ & $\mathrm{C}(27)-\mathrm{C}(25)-\mathrm{N}(2)-\mathrm{C}(11)$ & $-156.56(15)$ \\
\hline $\mathrm{C}(4)-\mathrm{C}(5)-\mathrm{C}(6)-\mathrm{C}(1)$ & $1.5(2)$ & $\mathrm{C}(1)-\mathrm{C}(11)-\mathrm{N}(2)-\mathrm{C}(26)$ & $-168.33(14)$ \\
\hline $\mathrm{C}(10)-\mathrm{C}(5)-\mathrm{C}(6)-\mathrm{C}(1)$ & $179.88(15)$ & $\mathrm{C}(1)-\mathrm{C}(11)-\mathrm{N}(2)-\mathrm{C}(25)$ & $55.7(2)$ \\
\hline $\mathrm{C}(4)-\mathrm{C}(5)-\mathrm{C}(6)-\mathrm{C}(7)$ & $-179.29(16)$ & $\mathrm{N}(4)-\mathrm{C}(13)-\mathrm{N}(3)-\mathrm{C}(12)$ & $-8.9(3)$ \\
\hline $\mathrm{C}(10)-\mathrm{C}(5)-\mathrm{C}(6)-\mathrm{C}(7)$ & $-0.9(2)$ & $\mathrm{C}(14)-\mathrm{C}(13)-\mathrm{N}(3)-\mathrm{C}(12)$ & $172.99(18)$ \\
\hline$C(2)-C(1)-C(6)-C(5)$ & $-2.8(2)$ & $\mathrm{C}(3)-\mathrm{C}(12)-\mathrm{N}(3)-\mathrm{C}(13)$ & $172.81(17)$ \\
\hline $\mathrm{C}(11)-\mathrm{C}(1)-\mathrm{C}(6)-\mathrm{C}(5)$ & $176.08(15)$ & $\mathrm{N}(3)-\mathrm{C}(13)-\mathrm{N}(4)-\mathrm{C}(17)$ & $-177.07(16)$ \\
\hline $\mathrm{C}(2)-\mathrm{C}(1)-\mathrm{C}(6)-\mathrm{C}(7)$ & $177.96(16)$ & $\mathrm{C}(14)-\mathrm{C}(13)-\mathrm{N}(4)-\mathrm{C}(17)$ & $1.0(3)$ \\
\hline $\mathrm{C}(11)-\mathrm{C}(1)-\mathrm{C}(6)-\mathrm{C}(7)$ & $-3.1(2)$ & $\mathrm{C}(16)-\mathrm{C}(17)-\mathrm{N}(4)-\mathrm{C}(13)$ & $0.9(3)$ \\
\hline $\mathrm{C}(6)-\mathrm{C}(5)-\mathrm{C}(10)-\mathrm{N}(1)$ & $-103.54(18)$ & $\mathrm{C}(18)-\mathrm{C}(17)-\mathrm{N}(4)-\mathrm{C}(13)$ & $-179.06(16)$ \\
\hline $\mathrm{C}(4)-\mathrm{C}(5)-\mathrm{C}(10)-\mathrm{N}(1)$ & $74.9(2)$ & $\mathrm{N}(1)-\mathrm{C}(19)-\mathrm{N}(5)-\mathrm{C}(23)$ & $178.31(15)$ \\
\hline $\mathrm{C}(2)-\mathrm{C}(1)-\mathrm{C}(11)-\mathrm{N}(2)$ & $-105.78(18)$ & $\mathrm{C}(20)-\mathrm{C}(19)-\mathrm{N}(5)-\mathrm{C}(23)$ & $0.4(2)$ \\
\hline $\mathrm{C}(6)-\mathrm{C}(1)-\mathrm{C}(11)-\mathrm{N}(2)$ & $75.3(2)$ & $\mathrm{C}(22)-\mathrm{C}(23)-\mathrm{N}(5)-\mathrm{C}(19)$ & $-2.3(2)$ \\
\hline $\mathrm{C}(4)-\mathrm{C}(3)-\mathrm{C}(12)-\mathrm{N}(3)$ & $91.1(2)$ & $\mathrm{C}(24)-\mathrm{C}(23)-\mathrm{N}(5)-\mathrm{C}(19)$ & $175.96(15)$ \\
\hline $\mathrm{C}(2)-\mathrm{C}(3)-\mathrm{C}(12)-\mathrm{N}(3)$ & $-87.8(2)$ & $\mathrm{O}(1)-\mathrm{C}(27)-\mathrm{O}(3)-\mathrm{C}(29)$ & $0.3(3)$ \\
\hline $\mathrm{N}(4)-\mathrm{C}(13)-\mathrm{C}(14)-\mathrm{C}(15)$ & $-2.4(3)$ & $\mathrm{C}(25)-\mathrm{C}(27)-\mathrm{O}(3)-\mathrm{C}(29)$ & $178.49(15)$ \\
\hline $\mathrm{N}(3)-\mathrm{C}(13)-\mathrm{C}(14)-\mathrm{C}(15)$ & $175.59(18)$ & $\mathrm{C}(31)-\mathrm{C}(29)-\mathrm{O}(3)-\mathrm{C}(27)$ & $-171.37(16)$ \\
\hline $\mathrm{C}(13)-\mathrm{C}(14)-\mathrm{C}(15)-\mathrm{C}(16)$ & $2.0(3)$ & $\mathrm{O}(2)-\mathrm{C}(28)-\mathrm{O}(4)-\mathrm{C}(30)$ & $-3.2(3)$ \\
\hline $\mathrm{C}(14)-\mathrm{C}(15)-\mathrm{C}(16)-\mathrm{C}(17)$ & $-0.3(3)$ & $\mathrm{C}(26)-\mathrm{C}(28)-\mathrm{O}(4)-\mathrm{C}(30)$ & $178.20(15)$ \\
\hline $\mathrm{C}(15)-\mathrm{C}(16)-\mathrm{C}(17)-\mathrm{N}(4)$ & $-1.2(3)$ & $\mathrm{C}(32)-\mathrm{C}(30)-\mathrm{O}(4)-\mathrm{C}(28)$ & $-176.99(17)$ \\
\hline $\mathrm{C}(15)-\mathrm{C}(16)-\mathrm{C}(17)-\mathrm{C}(18)$ & $178.70(18)$ & & \\
\hline $\mathrm{N}(5)-\mathrm{C}(19)-\mathrm{C}(20)-\mathrm{C}(21)$ & $1.6(3)$ & & \\
\hline
\end{tabular}


Table 5. Hydrogen bonds [ $\left[\AA\right.$ and $\left.{ }^{\circ}\right]$.

\begin{tabular}{lcccc}
\hline D-H...A & $\mathrm{d}(\mathrm{D}-\mathrm{H})$ & $\mathrm{d}(\mathrm{H} . . . \mathrm{A})$ & $\mathrm{d}(\mathrm{D} . . \mathrm{A})$ & $<(\mathrm{DHA})$ \\
& & & & \\
\hline $\mathrm{N}(1)-\mathrm{H}(01) \ldots \mathrm{N}(4) \# 1$ & $0.84(2)$ & $2.36(2)$ & $3.171(2)$ & $162.4(18)$ \\
$\mathrm{N}(3)-\mathrm{H}(03) \ldots \mathrm{O}(2) \# 2$ & $0.873(19)$ & $2.16(2)$ & $3.028(2)$ & $176.9(18)$ \\
\hline
\end{tabular}

Symmetry transformations used to generate equivalent atoms:

$\# 1-x+1,-y+1,-z$

$\# 2-x+1,-y+1,-z+1$ 